“C 2017 IEEE. Personal use of this material is permitted. Permission from IEEE must be obtained for all other uses, in any current or future media, including reprinting/republishing this material for advertising or promotional purposes, creating new collective works, for resale or redistribution to servers or lists, or reuse of any copyrighted component of this work in other works." 


\title{
Design of Multilayer SIW Cavity-Backed Slot Antenna Array
}

\author{
Yang Cai*, Yingsong Zhang*, Can Ding ${ }^{\dagger}$, Zuping Qian*, and Jie Liu* \\ *(Affiliation): PLA University of Science and Technology, Nanjing, China, \\ caiyang_1991@163.com \\ $\dagger$ (Affiliation): University of Technology, Sydney (UTS), Sydney, Australia \\ can.ding.1989@gmail.com
}

\begin{abstract}
In this paper, a multilayer substrate integrated waveguide (SIW) cavity-backed slot antenna array with wideband performance is proposed. In order to broaden the operating bandwidth of SIW slot antenna, multilayer cavities with gradually decreased permittivity and expanded aperture sizes are loaded above the slot, which realizes a smooth transition between SIW slot and free space. A wideband feeding network employing slot coupling is designed to excite the array elements. Results indicate the proposed array operates with $\mathbf{2 8 . 4 \%}$ bandwidth ranging from 22.4 to $29.8 \mathrm{GHz}$. Besides, stable broadside radiation patterns are obtained across the operating band.
\end{abstract}

Index Terms-multilayer, array, substrate integrated waveguide, slot antenna.

\section{INTRODUCTION}

In millimeter-wave band, substrate integrated waveguide (SIW) inherits merits from both microstrip lines and metal waveguides and exhibits advantages of easy fabrication, low transmission loss, and etc. Therefore, such electromagnetically closed planar waveguide structure has been employed as a good candidate for designing antenna arrays in millimeterwave band [1]. Cavity-backed slot antennas based on SIW have attracted many attentions and been proved to be useful in many applications.

As well known, SIW cavity-backed slot antenna has intrinsically narrow bandwidth. There have been several representative methods proposed in the past few years to enhance the bandwidth. Since the bandwidth of the cavity-backed slot antenna depended on the quality factor of the slot and cavity, the substrate under the slot was removed to broaden the bandwidth to $2.16 \%$ [2]. In [3], bandwidth of the SIW cavity-backed slot antenna could be enhanced to $6.3 \%$ by exciting hybrid SIW cavity modes. Through increasing the width to length ratio of the wide slot, dual resonances of SIW cavity-backed slot antenna were also generated and $11.6 \%$ bandwidth was achieved [4]. Besides, when the cavity-backed slot antenna was loaded with an open-ended cavity, the impedance bandwidth of a single element was enhanced to $23 \%$ [5].

As for designing cavity-backed slot antenna array, the feed network should be well builded to match the wideband performance of the array element. Despite of the excellent radiation performance, the bandwidths of series-feed and shunt-feed arrays were restricted since the radiators and feed network shared the same layer [6]-[8]. In our previous work, a novel
SIW cavity-backed slot antenna array without individual feed network was designed and $14 \%$ wide impedance bandwidth was obtained [9]. Based on the novel subarray, a largescale corporate-feed antenna array with $15 \%$ bandwidth was presented [10]. Besides, an SIW higher order mode cavitybacked slot antenna array with simplified feed network was presented in [11]. The slots were arranged asymmetrically in the cavity to introduce new resonances and $16.7 \%$ bandwidth was obtained. When a larger bandwidth is further required, the state-of-art technologies fail to achieve breakthrough in broadening bandwidth due to the resonant characteristic of the slot element.

In this paper, a 16-element multilayer SIW cavity-backed slot antenna array is presented. The array element can be viewed as a modified open-ended cavity with variations both in aperture size and permittivity, which acts as a transition from the SIW cavity-backed slot to the air. The remainder of the paper is organized into three additional sections. In Section II, the feed network and a four-element subarray are specifically introduced. A 16-element array are presented and the simulated results are shown in Section III. Finally, the conclusion is drawn in Section IV.

\section{SUbARRAY DESIGN}

Since array configuration shows much reliance on the feed network, a four-way power divider for feeding a subarray is specifically introduced, following by showing the performance of a four-element subarray. Based on the designing procedure, the array scale can be simply enlarged with increasing the scale of the feed network. The array is designed with the aid of High Frequency Structure Simulator (HFSS).

\section{A. 1-to-4 Power Divider}

Fig. 1 depicts the configuration of the four-way power divider. The power divider consists of two layers with heights of $h_{1}$ and $h_{2}$, respectively. The energy is input from port 1 in bottom layer and coupled through the slot with size of $l_{r} \times w_{r}$ with spacings $d_{x 2}$ and $d_{y 2}$ to the endwall and sidewall, respectively. An adjusting via with spacings $d_{x 1}$ and $d_{y 1}$ to the endwall and sidewall is adopted to further realize good coupling effect. When the energy arrives at the top layer of the feed network, a coupling window with size of $l_{g} \times w_{g}$ is opened to distribute the energy equally in four ways. An adjusting via 


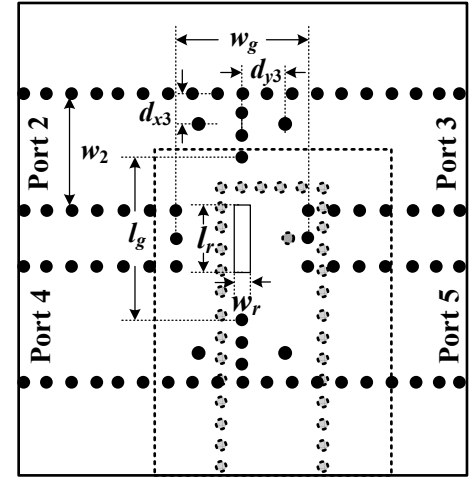

(a)

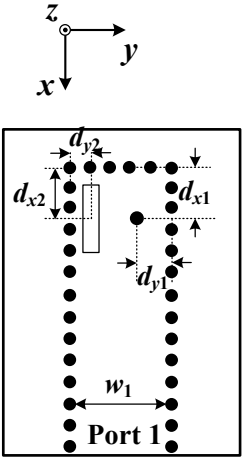

(b)
Fig. 1. Configuration of the 1-to-4 power divider. (a) Perspective view of the power divider. (b) Top view of the bottom layer.

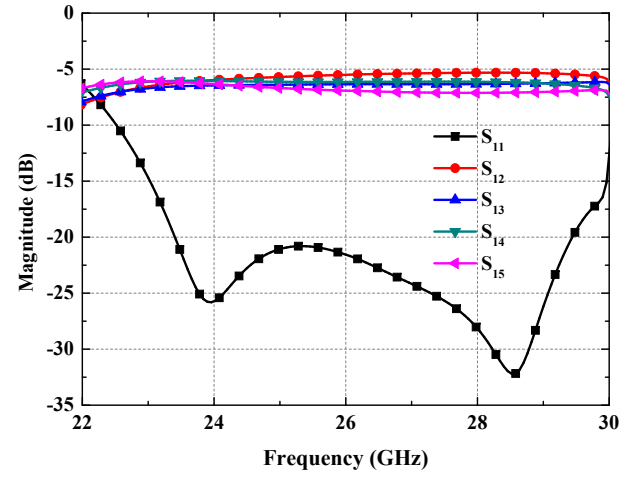

(a)

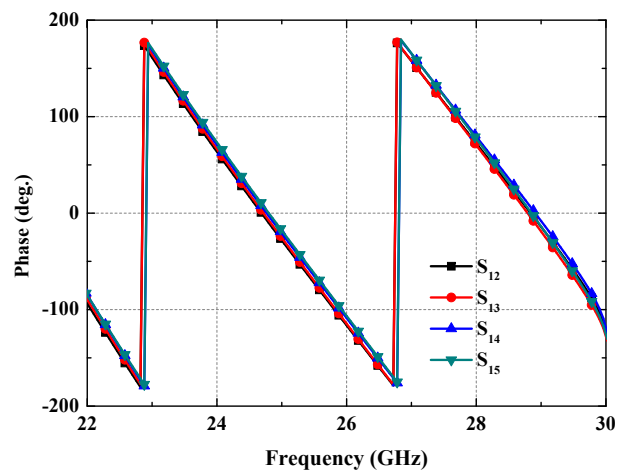

(b)

Fig. 2. Simulated results of the 1-to-4 power divider. (a) S-parameters. (b) Phases.

is placed with spacings $d_{x 3}$ and $d_{y 3}$ to the corner to realize good transition from the coupling window to the sub-channels.

The results of the power divider are calculated in Fig. 2 with the parameters listed in Table I. In the simulated band ranging from 22.5 to $30 \mathrm{GHz}$, the reflection coefficients are below $10 \mathrm{~dB}$. Moreover, within this simulated band, the magnitude difference is less than $2.5 \mathrm{~dB}$ while phase error of the four outputs is less than 12 degrees.
TABLE I

PARAMETERS OF THE POWER DIVIDER. (UNIT: MM).

\begin{tabular}{c|c|c|c}
\hline \hline Symbol & Quantity & Symbol & Quantity \\
\hline$d_{x 1}$ & 3 & $w_{1}$ & 6.5 \\
$d_{y 1}$ & 2.2 & $w_{2}$ & 7 \\
$d_{x 2}$ & 1.3 & $w_{g}$ & 8 \\
$d_{y 2}$ & 3 & $l_{g}$ & 10 \\
$d_{x 3}$ & 1.6 & $w_{r}$ & 1.2 \\
$d_{y 3}$ & 2.5 & $l_{r}$ & 5.5 \\
$h_{1}$ & 0.5 & $h_{2}$ & 1 \\
\hline \hline
\end{tabular}
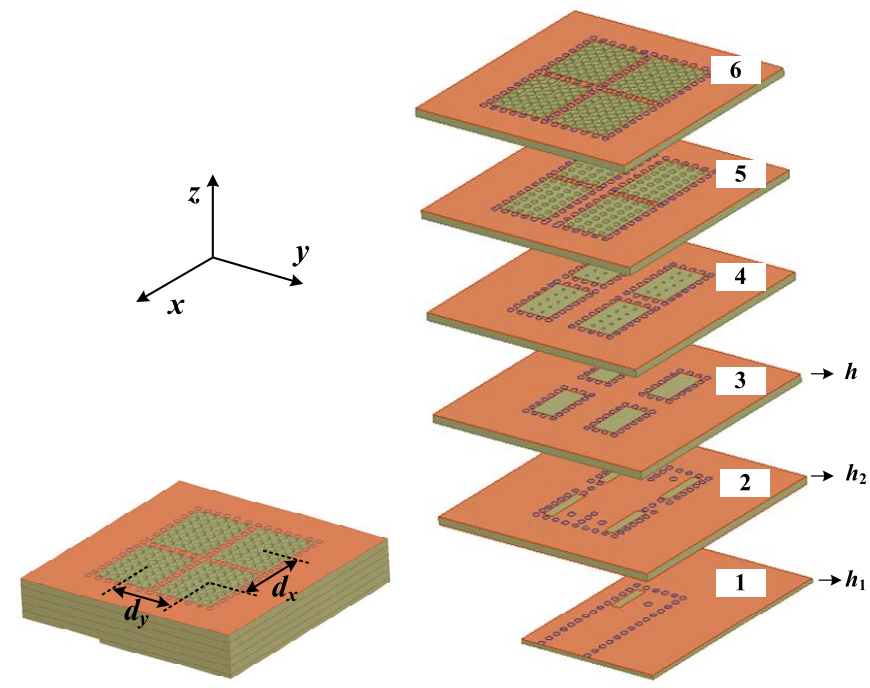

(a)

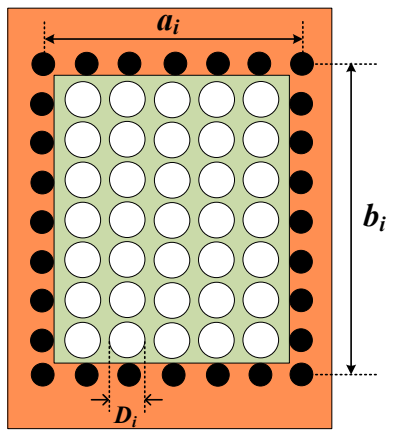

(b)

Fig. 3. Configuration of the four-element subarray. (a) Whole and anatomy views. (c) Cavity cell.

\section{B. Design of Four-Element Subarray}

Since the outputs of the four-way power divider are almost in-phase, array elements can be placed symmetrically with related to the centre of the power divider. The geometrical configuration of the four elements subarray is depicted in Fig. 3. This subarray is made by six layers and the feed network occupies the bottom two layers. In order to excite the horn elements, the four-way power divider is shorted by metallized vias to form a cavity and four slots are placed at the corners of the cavity to convert the vertically polarized wave in the power 
TABLE II

PARAMETERS OF THE SUBARRAY. (UNIT: MM).

\begin{tabular}{c|c|c|c}
\hline \hline Symbol & Quantity & Symbol & Quantity \\
\hline$a_{1}$ & 3.1 & $D_{1}$ & 0 \\
$b_{1}$ & 6.5 & $D_{2}$ & 0.4 \\
$a_{2}$ & 4.7 & $D_{3}$ & 0.9 \\
$b_{2}$ & 8.1 & $D_{4}$ & 1.2 \\
$a_{3}$ & 6.3 & $d_{x}$ & 10.3 \\
$b_{3}$ & 9.7 & $d_{y}$ & 9.2 \\
$a_{4}$ & 7.9 & $h$ & 1 \\
$b_{4}$ & 9.7 & $d$ & 1.6 \\
\hline \hline
\end{tabular}

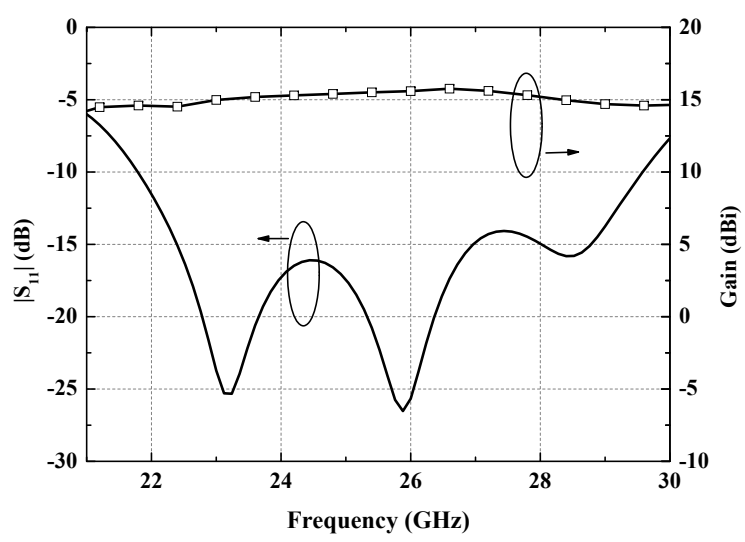

Fig. 4. Simulated reflection coefficients and broadside gains of the fourelement array.

divider into the horizontally polarized wave in the multilayer cavities. The cavities, made by four PCB boards with a same height $h$, are directly placed above the slots. The aperture size of a cavity cell in the $i$ th layer is $a_{i} \times b_{i}$ and air vias with diameter of $D_{i}$ are perforated in the cavity to manipulate the permittivity. The cavity walls are made by the surrounded metallized vias with diameter of $d$. The element spacings in $\mathrm{x}$ - and y-direction are defined as $d_{x}$ and $d_{y}$.

A four-element subarray is designed and simulated with the parameters listed in Table II. As shown in Fig. 4, this subarray owns $30.6 \%$ impedance bandwidth in the frequency range from $21.8 \mathrm{GHz}$ to $29.7 \mathrm{GHz}$ with $\left|S_{11}\right|$ below $-10 \mathrm{~dB}$. In this band, a flat gain response fluctuant around $15 \mathrm{dBi}$ is obtained. Besides, stable broadside radiation patterns can be observed in Fig. 5. By the way, when the operating frequency goes high to $29.7 \mathrm{GHz}$, a relatively high side lobe level is resulted, which mainly comes from the increased element spacings in comparison with the operating wavelength.

\section{ARray Design And Results}

The four-element subarray can be easily extended to a 16element array when an $\mathrm{H}$-shaped power divider is employed, as shown in Fig. 6. A prototype is fabricated and the photograph is shown in Fig. 7. A transition from microstrip line to SIW is designed and a connecting device is employed to facilitate the measurement of antenna performance. All the prototypes

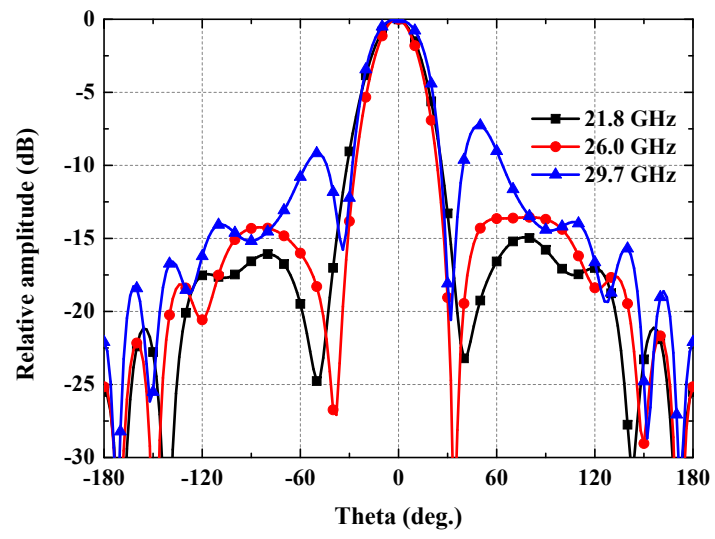

(a)

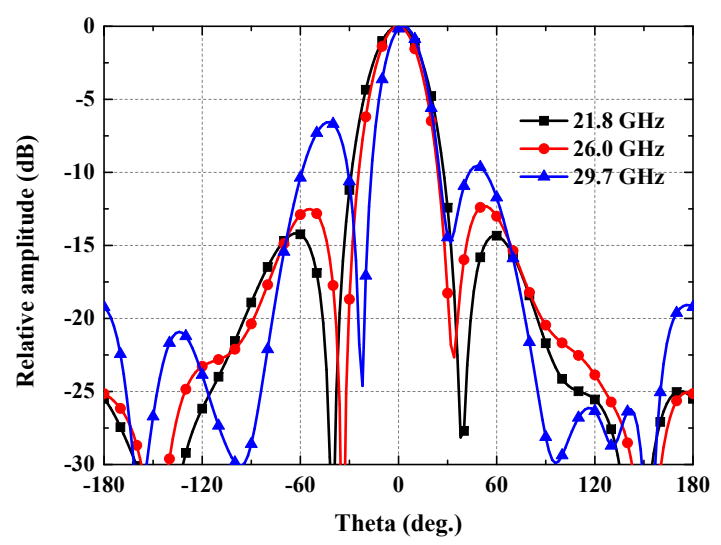

(b)

Fig. 5. Simulated radiation patterns of the four-element array at three representing frequencies. (a) E-plane. (b) H-plane.

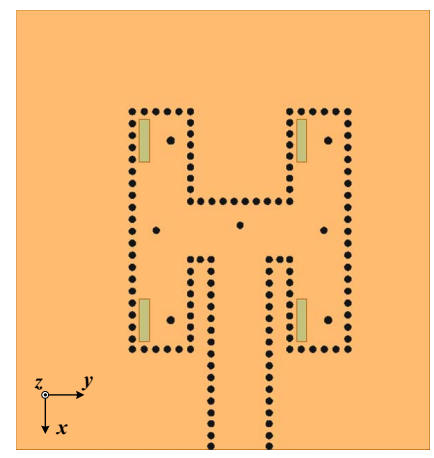

(a)

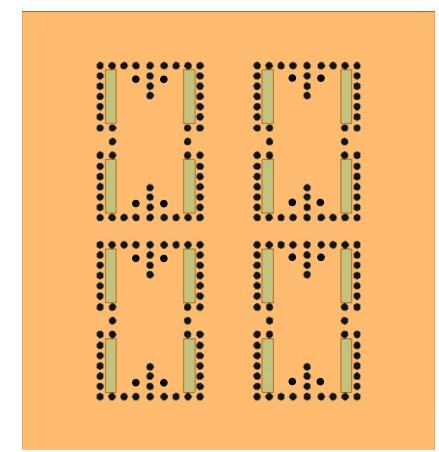

(b)
Fig. 6. Configuration of the feed network for the proposed array. (a) Layer 1. (b) Layer 2.

are fabricated using Rogers RT/Duroid 5880 PCB boards with permittivity of 2.2 and loss tangent of 0.0009 .

The simulated reflection coefficients and broadside gains are calculated in Fig. 8. It can be observed that in the band ranging from 22.4 to $29.8 \mathrm{GHz}$, the simulated $\left|S_{11}\right| \mathrm{s}$ are below $-10 \mathrm{~dB}$ and the relative impedance bandwidth of the designed array reaches to $28.4 \%$. Besides, the simulated broadside gains are also calculated in this figure. A flat gain response fluctuant around $20 \mathrm{dBi}$ with up to $2 \mathrm{dBi}$ gain variation is obtained. The far-field radiation patterns at three representing frequencies 


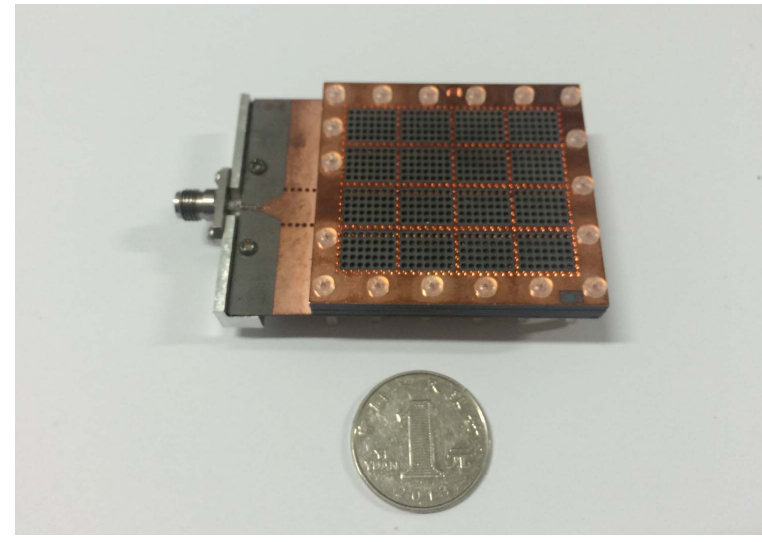

Fig. 7. Photograph of the fabricated prototype.

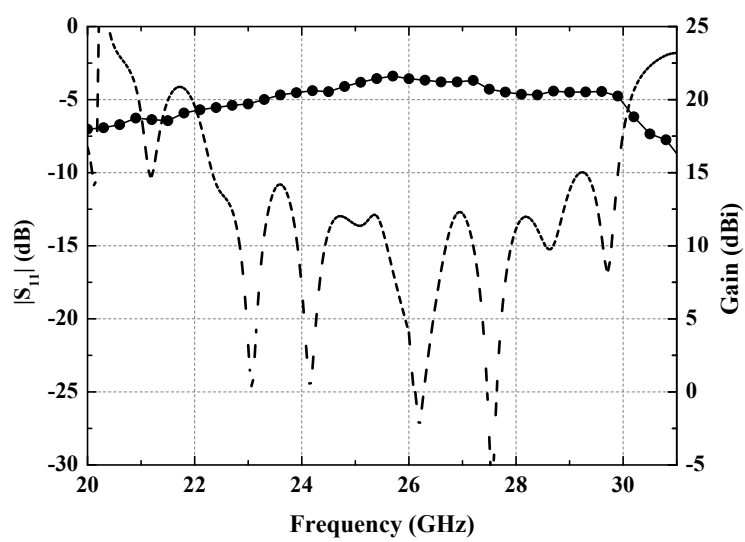

Fig. 8. Simulated reflection coefficients and gains of the proposed array.

in the operating band are plotted in Fig. 9. The simulated side lobe level is typically below $-10 \mathrm{~dB}$, and stable broadside radiation pattern is obtained within the operating band.

\section{CONCLUSION}

In the paper, a multilayer SIW horn antenna array with wideband performance is proposed. Through loading multilayer horn cavities with both gradually increased aperture sizes and decreased permittivity, a smooth transition is realized between the SIW cavity-backed slot antenna and the free space. Moreover, considering the extensibility of the array, a dual-layer corporate-feed network based on slot-coupling is employed for feeding the array elements. Results indicate the proposed antenna array achieves up to $28.4 \%$ operating bandwidth with stable broadside radiation patterns across the whole operating band.

\section{ACKNOWLEDGMENTS}

This work was supported in part by the National Science Foundation of China under Grant 61271103, and in part by the Open Project of State Key Laboratory of Millimeter Waves, Southeast University China under Grant K201505.

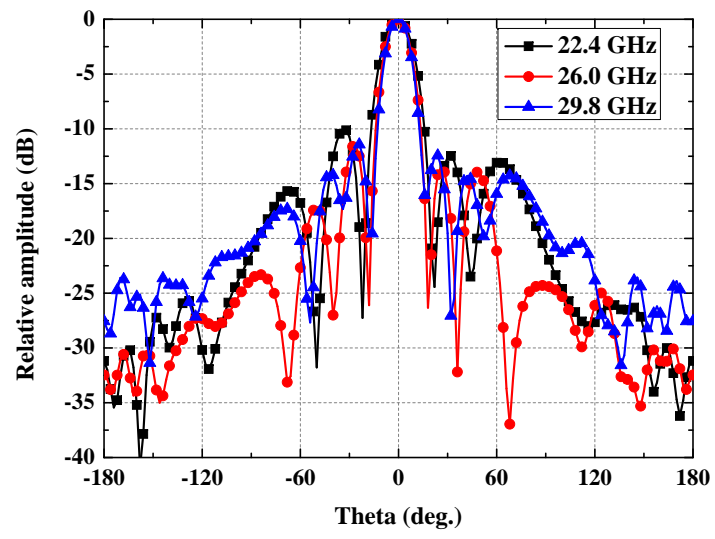

(a)

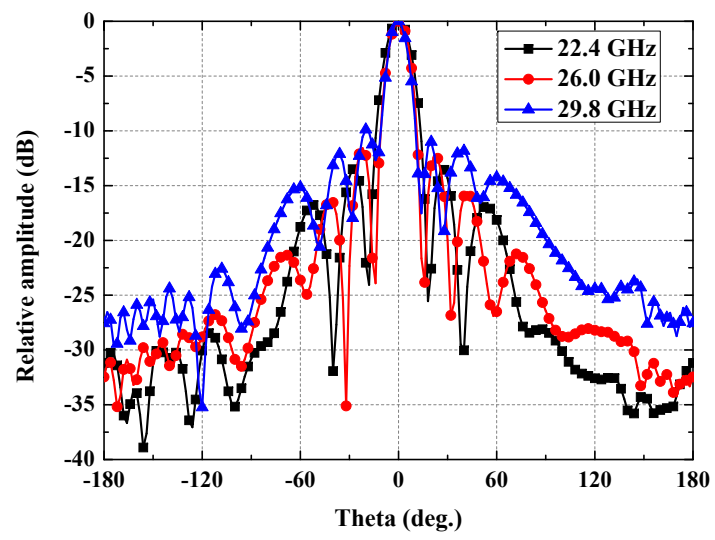

(b)

Fig. 9. Simulated radiation patterns of the proposed array at three representing frequencies. (a) E-plane. (b) H-plane.

\section{REFERENCES}

[1] K. Wu, Y.-J. Cheng, T. Djerafi, and W. Hong, "Substrate-integrated millimeter-wave and terahertz antenna technology," Proceedings of the IEEE, vol. 100, no. 7, pp. 2219-2232, Jul. 2012.

[2] S.Yun, D. Kim, and S.Nam, "Bandwidth and efficiency enhancement of cavity-backed slot antenna using a substrate removal," IEEE Antennas Wireless Propag. Lett., vol. 11, pp. 1458-1461, 2012.

[3] G.-Q. Luo, Z.-F. Hu, W.-J. Li, X.-H. Zhang, L.-L. Sun, and J.-F. Zheng, "Bandwidth-enhanced low-profile cavity-backed slot antenna by using hybrid SIW cavity modes," IEEE Trans. Antennas Propag., vol. 60, no. 4, pp. 1698-1704, Jan. 2012.

[4] K. Gong, Z.-N. Chen, X.-M. Qing, Z. Song, P. Chen, and W. Hong, "Empirical formula of cavity dominant mode frequency for $60-\mathrm{GHz}$ cavity backed wide slot antenna," IEEE Trans. Antennas Propag., vol. 61, no. 2, pp. 1698-1704, Feb. 2013.

[5] J.-F. Xu, Z.-N. Chen, X.-M. Qing, and W. Hong, "Bandwidth enhancement for a $60 \mathrm{GHz}$ substrate integrated waveguide fed cavity array antenna on LTCC," IEEE Trans. Antennas Propag., vol. 59, no. 3, pp. 826-832, Mar. 2011.

[6] X. Chen, K. Wu, L. Han, and F.-F.He, "Low-cost high gain planar antenna array for $60-\mathrm{GHz}$ band applications," IEEE Trans. Antennas Propag., vol. 58, no. 6, pp. 2126-2129, Jun. 2010.

[7] Y. Zhang, Z.-N. Chen, X.-M. Qing, and W. Hong, "Wideband millimeterwave substrate integrated waveguide slotted narrow-wall fed cavity antennas," IEEE Trans. Antennas Propag., vol. 59, no. 5, pp. 1488-1496, May 2011.

[8] K. Gong, Z.-N. Chen, X.-M. Qing, P. Chen, and W. Hong, "Substrate integrated waveguide cavity-backed wide slot antenna for $60-\mathrm{GHz}$ bands," IEEE Trans. Antennas Propag., vol. 60, no. 12, pp. 6023-6026, Dec. 2012.

[9] D.-F. Guan, Z.-P. Qian, Y.-S. Zhang, and Y. Cai, "Novel SIW cavity 
backed antenna array without using individual feeding network," IEEE Antennas Wireless Propag. Lett., vol. 13, pp. 423-426, 2014.

[10] D.-F. Guan, C. Ding, Z.-P. Qian, Y.-S. Zhang, W.-Q. Cao, and E. Dutkiewicz, "An SIW based large-scale corporate-feed array antenna," IEEE Trans. Antennas Propag., vol. 63, no. 7, pp. 2969-2976, Jul. 2015.

[11] P. Wu, S. Liao, and Q. Xue, "A substrate integrated slot antenna array using simplified feeding network based on higher order cavity modes," IEEE Trans. Antennas Propag., vol. 64, no. 1, pp. 126-135, Jan. 2016. 